\title{
Tumores primarios de sacro: análisis de resultados y complicaciones
}

\author{
García-Ortega DY,* Clara-Altamirano MA,** Gómez-Pedraza A, *** Martínez-Said H,* \\ Maciel-Miranda A, ${ }^{* * * *}$ Caro-Sánchez CHS, ${ }^{* * * * *}$ Cuellar-Hubbe M, ${ }^{* * * * * *}$ Partida-Nava GV******* \\ Instituto Nacional de Cancerología de México
}

RESUMEN. Introducción: Los tumores primarios de sacro son poco frecuentes, el tratamiento en la mayoría de los casos es quirúrgico con o sin radioterapia adyuvante; los resultados oncológicos y funcionales suelen ser adversos con una tasa alta de complicaciones. Material y métodos: Se realizó un estudio retrospectivo, analítico y observacional que incluye 22 casos tratados entre el 2000 y 2017, se analizaron sus características demográficas, el tipo de tratamiento en la recidiva y el resultado oncológico y funcional; asimismo, se analizó la tasa de complicaciones. Resultados: 22 pacientes fueron sometidos a sacrectomía, con abordaje posterior por cordoma (13 pacientes), tumor de células gigantes (tres pacientes) y otros (seis pacientes). Se realizaron tres sacrectomías totales, 13 sacrectomías parciales, dos hemisacrectomías y cuatro sacrectomías ampliadas. La media de duración quirúrgica fue de $\mathbf{2 2 9}$ minutos, con un sangrado promedio de $2,100 \mathrm{~cm}^{3}$, el tamaño tumoral promedio fue de $13.8 \mathrm{~cm}(6-30 \mathrm{~cm})$; presentaron complicaciones 10 pacientes, ocho por infección de sitio quirúrgico, uno por hernia sacra y uno por osteomielitis. La supervivencia global fue de 44.4 meses. Discusión: El tratamiento de los tumores sacros es complejo y requiere un equipo multidisciplinario; el resultado oncológico es adecuado cuando
ABSTRACT. Introduction: Primary sacral tumors are rare, treatment in most cases is surgical with or without adjuvant radiotherapy; oncology and functional results are usually adverse with a high rate of complications. Material and methods: We conducted a retrospective, analytical and observational studies that includes 22 cases treated between 2000 and 2017, analyzed their characteristics demographic, the type of treatment received, and the oncological, functional results and the rate of complications were analized. Results: 22 patients were subjected to sacrectomy with posterior approah because of cordoma (13 patients), giant cell tumour (three patients) and other (six patients). Three total sacrectomies, 13 partial sacrectomies, two hemisacrectomies and four sacrectomies enlarged were performed. The mean surgical time was 229 minutes, with an average bleeding of $2,100 \mathrm{~cm}^{3}$, the average tumour size was $13.8 \mathrm{~cm}(6-30 \mathrm{~cm}) ; 10$ patients were presented with complications, eight by surgical site infection, one sacral hernia and one osteomyelitis. Overall survival was $\mathbf{4 4 . 4}$ months. Conclusions: Treatment of sacral tumors is complex, requires a multidisciplinary team; the oncological result is adequate when you get free margins of neoplasia,

Nivel de evidencia: IV

\footnotetext{
* Cirujano Oncólogo. Adscrito, Departamento de Piel y Partes Blandas.

** Ortopedista Oncólogo. Adscrito, Departamento de Piel y Partes Blandas.

*** Cirujano Oncólogo. Adscrito al Departamento de Cabeza y Cuello.

**** Cirujano Plástico. Adscrito, Departamento de Piel y Partes Blandas.

***** Patólogo Oncólogo. Adscrito, Departamento de Piel y Partes Blandas.

****** Cirujano Oncólogo. Jefe de Departamento de Piel y Partes Blandas.

******* Cirujano Oncólogo.

Instituto Nacional de Cancerología.

Dirección para correspondencia:

Dorian Yarih García-Ortega

Cirujano Oncólogo. Adscrito, Departamento de Piel y Partes Blandas, Instituto Nacional de Cancerología.

Av. San Fernando Núm. 22, Col. Sección XVI, Alcaldía Tlalpan, C.P. 14080, CDMX.

Tel: 56280400 y 56554766 ,

E-mail: dr_doriangarcia@me.com
}

Este artículo puede ser consultado en versión completa en http://www.medigraphic.com/actaortopedica 
se consiguen márgenes libres de neoplasia, los resultados funcionales estarán determinados por el tipo de resección y la tasa de complicaciones es alta; sin embargo, es la mejor alternativa de curación.

Palabras clave: Sacrectomía, complicaciones, resultados a largo plazo.

\section{Introducción}

Los tumores sacros son raros, condicionan 1-7\% del total de los tumores ocurridos en la columna vertebral. ${ }^{1}$ En términos globales, la respuesta a otras modalidades como la quimioterapia y la radioterapia es deficiente, por lo que la mejor alternativa de curación es la quirúrgica; siendo la sacrectomía y sus variantes el tratamiento de elección. La compleja región anatomía en la que se asientan estos tumores hace que sea un reto quirúrgico con una alta tasa de

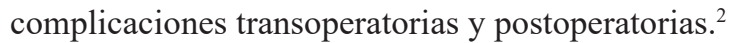

El diagnóstico de estos tumores es complicado y habitualmente tardío, debido a que el crecimiento de éstos es lento e indolente con frecuencia hacia la cavidad pélvica, a la exploración física suele no encontrarse ninguna anomalía; el diagnóstico ocurre a menudo de forma incidental o cuando está muy avanzada la neoplasia primaria y genera síntomas secundarios a la compresión o invasión de las estructuras nerviosas que pueden ir desde dolor hasta incontinencia fecal, urinaria o mixta. ${ }^{3}$

La resección quirúrgica tiende a ser el tratamiento de elección y el que mejor tasa de curación genera; sin embargo, la sacrectomía es un procedimiento de una alta demanda quirúrgica que requiere un equipo altamente capacitado, debido a la potencial lesión de estructuras que circundan la región sacra y que limitan el espacio quirúrgico y dificultan la obtención de márgenes quirúrgicos adecuados. ${ }^{2}$

Las sacrectomías son clasificadas de acuerdo al nivel de resección y el sacrificio de los nervios sacros en totales o parciales. La obtención de márgenes quirúrgicos negativos es fundamental para incrementar los períodos libres de enfermedad y con esto disminuir las recurrencias locales. ${ }^{4} \mathrm{Sin}$ embargo, para llegar al objetivo de márgenes adecuados en la gran mayoría de las ocasiones será necesario el sacrificio de nervios sacros, con las secuelas subsecuentes que impactarán en la calidad de vida del paciente, éstas pueden ser alteraciones funcionales intestinales, incontinencia fecal, incontinencia urinaria o mixta, disfunción sexual; en muchos de los casos los pacientes requerirán colostomías temporales o definitivas y derivaciones urinarias.

La incidencia de complicaciones quirúrgicas va de 40$60 \%$ de los casos e incluyen infección de sitio quirúrgico, dehiscencia de las heridas, presencia de hernias sacras que condicionan postoperatorios marcadamente complicados con estancias intrahospitalarias largas y múltiples reintervenciones para resolverlas. ${ }^{4}$ the functional results will be determined by the type of resection, and the rate of complications is high however is the best alternative healing in our hospital.

Key words: Sacrectomy, complications, long term results.

Posteriormente a la resección un porcentaje alto de los pacientes requerirán algún tratamiento adyuvante, principalmente radioterapia y en algunos casos quimioterapia y radioterapia concomitante.

El propósito de este estudio es presentar los resultados oncológicos y las complicaciones en los pacientes tratados en el Instituto Nacional de Cancerología de pacientes con tumores originados en el sacro.

\section{Material y métodos}

Se realizó un estudio retrospectivo, analítico y observacional de los pacientes tratados en el Instituto Nacional de Cancerología de México con diagnóstico de tumor maligno primario de sacro en el período comprendido de Enero de 2000 al 31 de Diciembre de 2017, se excluyeron todos aquellos pacientes con tumores benignos, tumores metastásicos al sacro, así como todos aquellos pacientes que tenían expedientes clínicos incompletos o abandonaron el seguimiento. Todos los pacientes fueron sometidos a algún tipo de sacrectomía con abordaje posterior únicamente. La decisión del tratamiento adyuvante fue tomada por un equipo multidisciplinario, el seguimiento fue realizado de forma convencional a través de consultas cada tres a cuatro meses durante los primeros dos años, cada seis meses hasta los cinco años y posteriormente cada año, se hicieron estudios de imagen de control cada seis meses, TAC de pelvis y estudio de imagen de tórax.

Se conformó una base de datos que incluía variables demográficas (edad, sexo, etc.) tipo histológico del tumor primario, tipo de tratamiento preoperatorio, nivel de resección, márgenes, reconstrucción, resultados funcionales (incluyendo motor y sensitivo, así como presencia de incontinencia), tratamiento adyuvante, complicaciones, período libre de enfermedad y supervivencia global.

La información recabada fue analizada con el programa estadístico SPSS v. 22, se obtuvo para variables cuantitativas medidas de tendencia central y dispersión. Para las variables cualitativas se emplearon frecuencias y porcentajes. Se utilizaron curvas de Kaplan-Meier para supervivencia.

\section{Resultados}

Durante el período de 2000 a 2017 se trataron 22 pacientes en el Instituto Nacional de Cancerología de México, 13 fueron hombres (59.1\%) y nueve mujeres $(40.9 \%)$, 
con una mediana de edad de 44 años (de 21 a 84 años), el tipo histológico más frecuente fue cordoma en 13 pacientes $(59.1 \%)$ seguido de tumor de células gigantes en tres casos (13.6\%) y otros como tumor neuroectodérmico primitivo, meduloblastoma, condrosarcoma y osteosarcoma (Tabla 1). El síntoma que más frecuentemente se asoció fue dolor en seis pacientes $(30 \%)$, incontinencia fecal y urinaria en un caso $(5 \%)$, parestesias en un caso $(5 \%)$, siete pacientes presentaron más de un síntoma, incontinencia fecal, urinaria o mixta, dolor o parestesias y en dos casos el diagnóstico fue incidental, por lo que no presentaban ningún síntoma.

18 pacientes fueron sometidos a tratamiento quirúrgico inicial, tres fueron sometidos a quimioterapia y un caso a quimioterapia/radioterapia concomitante. El tipo de resec-

\begin{tabular}{|c|c|}
\hline Características & $\mathrm{n}(\%)$ \\
\hline \multicolumn{2}{|l|}{ Sexo } \\
\hline Masculino & $13(59.1)$ \\
\hline Femenino & $9(40.9)$ \\
\hline \multicolumn{2}{|l|}{ Síntomas al diagnóstico } \\
\hline Dolor & $6(30)$ \\
\hline Incontinencia & $1(5)$ \\
\hline Mixto & $7(35)$ \\
\hline Ninguno & $2(10)$ \\
\hline \multicolumn{2}{|l|}{ Tipo histológico } \\
\hline Cordoma & $13(59.1)$ \\
\hline Tumor de células gigantes & $3(13.6)$ \\
\hline Tumor germinal & $1(5.0)$ \\
\hline PNET* $^{*}$ & $1(5.0)$ \\
\hline Osteosarcoma & $1(5.0)$ \\
\hline Condrosarcoma & $1(5.0)$ \\
\hline Meduloblastoma & $1(5.0)$ \\
\hline Tumor fibroso solitario & $1(5.0)$ \\
\hline \multicolumn{2}{|l|}{ Tipo de sacrectomía } \\
\hline Total & $3(10)$ \\
\hline Parcial & $13(55)$ \\
\hline Ampliada & $4(25)$ \\
\hline Hemisacrectomía & $2(10)$ \\
\hline \multicolumn{2}{|l|}{ Extensión } \\
\hline L5 & $3(13.6)$ \\
\hline S1 & $7(31.8)$ \\
\hline $\mathrm{S} 2$ & $7(31.8)$ \\
\hline S3 & $5(22.7)$ \\
\hline \multicolumn{2}{|l|}{ Márgenes } \\
\hline R0 & $12(54.5)$ \\
\hline $\mathrm{R} 1$ & $10(45.5)$ \\
\hline \multicolumn{2}{|l|}{ Radioterapia adyuvante } \\
\hline Sí & $9(40.9)$ \\
\hline No & $13(59.1)$ \\
\hline \multicolumn{2}{|l|}{ Recurrencia } \\
\hline Sí & $6(27.0)$ \\
\hline No & $16(73.0)$ \\
\hline \multicolumn{2}{|l|}{ Complicaciones } \\
\hline Infección del sitio quirúrgico & $8(40)$ \\
\hline Hernia sacra & $1(5)$ \\
\hline Osteomielitis & $1(5)$ \\
\hline
\end{tabular}

ción fue sacrectomía parcial en 13 casos, total en tres, hemisacrectomía en tres y cuatro casos sacrectomía ampliada; la extensión de la resección fue en tres casos a nivel de L5, $\mathrm{S} 1$ en siete casos, $\mathrm{S} 2$ en siete casos y cinco casos en $\mathrm{S} 3$. El tiempo quirúrgico promedio fue de 229 minutos (120360 minutos), el sangrado promedio fue de $2,100 \mathrm{~cm}^{3}(80-$ $6,000)$; el tamaño promedio del tumor fue de $13.8 \mathrm{~cm}(6-30$ $\mathrm{cm})$, se realizó colostomía terminal en siete casos y una derivación urinaria, ocho pacientes presentaron incontinencia mixta y los pacientes a los que no se realizó derivación urinaria se capacitó para realizar sondeo vesical intermitente.

Se presentó un total de 10 complicaciones mayores, ocho infecciones de sitio quirúrgico, una osteomielitis, que fue manejada con aseo quirúrgico y antimicrobianos, y una hernia sacra, manejada de forma conservadora.

Los márgenes de resección fueron negativos en 12 pacientes, positivos microscópicos en 10 casos; nueve pacientes recibieron radioterapia adyuvante. Seis casos presentaron recurrencia, de ésta, cuatro locales y dos a distancia, el seguimiento fue de 45.35 meses (1-153), la supervivencia global fue de 44.4 meses, cinco pacientes murieron a causa de tumor primario. Se encontró asociación estadística entre el margen de resección, el período libre de enfermedad $(\mathrm{p}=0.03)$ y la supervivencia global, respectivamente (Figuras 1 y 2).

\section{Discusión}

La resección quirúrgica en los tumores primarios de sacro es el tratamiento que ha demostrado los mejores resultados, debido a que éstos tienden a ser quimiorresistentes y radiorresistentes. ${ }^{2}$

Desafortunadamente, el tratamiento está asociado a una serie de efectos secundarios a la resección de las fibras nerviosas sacras resultando en incontinencia fecal, urinaria e impotencia con un claro impacto en la calidad de vida del pa-

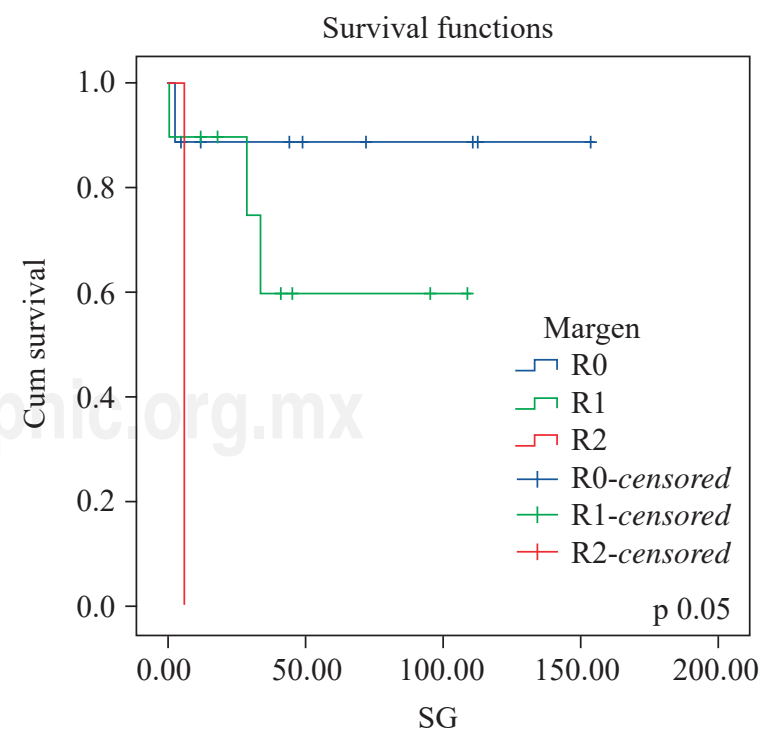

Figura 1: Tablas de supervivencia global, período libre de enfermedad y márgenes. 


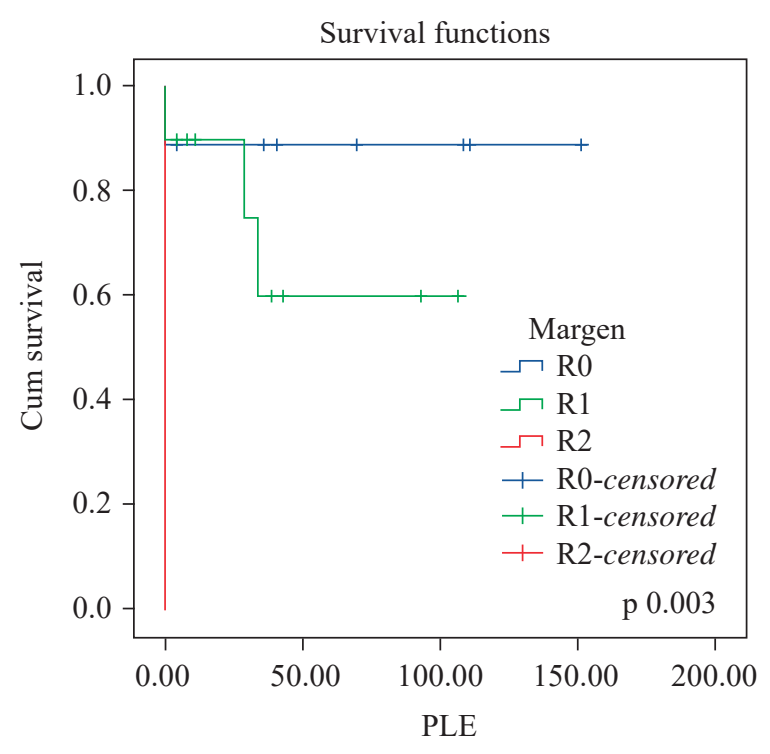

Figura 2: Tablas de supervivencia global, supervivencia global y márgenes.

ciente, ${ }^{2,3,4,5,6,7,8}$ tal y como presentamos en este estudio el número de pacientes que tienen como secuela esto es muy alto.

El predictor más importante de supervivencia global y recurrencia tanto local como a distancia sin importar la histología es la presencia de márgenes negativos, siendo la única variable con asociación estadística en nuestro estudio. Debemos tomar en cuenta, siempre, que la resección completa en este grupo de pacientes tiende a ser complicada por múltiples factores tales como el tamaño tumoral y la relación neurovascular del sacro.

El conocimiento detallado de la anatomía, así como la elección adecuada del paciente y el procedimiento quirúrgico por un equipo multidisciplinario, es determinante en el éxito del tratamiento con un impacto en la calidad de vida, la supervivencia global y período libre de enfermedad.

La tasa de complicaciones postoperatorias después del tratamiento multimodal (principalmente cirugía más radioterapia postoperatoria) es alto y oscila entre 32-67\% según la serie que se revise, en nuestro caso no es diferente con una tasa de complicaciones de 50\%. De todas las complicaciones la más frecuente es la infección de sitio quirúrgico similar a lo encontrado en nuestra serie de casos. ${ }^{9,10,11,12}$ Creemos que dentro de los factores que podrían mejorar la presencia de infecciones de sitio quirúrgico se encuentra la realización de colostomías en asa de forma electiva a pesar de que el sitio de resección no sea alto, esto con la finalidad de evitar la contaminación de la herida quirúrgica y posteriormente en aquellos pacientes que tengan adecuada función de los esfínteres poder reinstalar su tránsito intestinal normal. Evidentemente, existen otros factores asociados a la presencia de éstos entre los que se incluyen el estado funcional, estado nutricional, exposición a tratamientos previos como la quimioterapia y radioterapia. ${ }^{13}$

\section{Conclusiones}

A pesar de la alta tasa de complicaciones y efectos secundarios de un manejo agresivo multimodal para los pacientes con tumores malignos primarios de sacro, consideramos que es la mejor opción de cura que provee períodos libres de enfermedad y supervivencias globales mayores. Sin embargo, la toma de decisiones sobre el tratamiento tiene que ser desarrollada por un equipo multimodal; una resección incompleta impacta directamente en el pronóstico de estos pacientes, por lo que debe ser realizada por cirujanos experimentados.

Bibliografía

1. Feldenzer JA, McGauley JL, McGillicuddy JE. Sacral and presacral tumors: problems in diagnosis and management. Neurosurgery. 1989; 25(6): 884-91.

2. Fourney DR, Rhines LD, Hentschel SJ, Skibber JM, Wolinsky JP, Weber KL, et al. En bloc resection of primary sacral tumors: classification of surgical approaches and outcome. J Neurosurg Spine. 2005; 3(2): 111-22.

3. Todd L, Yaszemski M, Currier B, Fuchs B, Kim C, Sim F. Bowel and bladder function after major sacral resection. Clin Orthop Relat Res. 2002; 397: 36-9.

4. Horan TC, Gaynes RP, Martone WJ, Jarvis WR, Emori TG. CDC definitions of nosocomial surgical site infections, 1992: a modification of CDC definitions of surgical wound infections. Infect Control Hosp Epidemiol. 1992; 13(10): 606-8.

5. Chen KW, Yang HL, Lu JY, Liu J, Chen X. Prognostic factors of sacral chordoma after surgical therapy: a study of 36 patients. Spinal Cord. 2010; 48(2): 166-71.

6. Biagini R, Ruggieri P, Mercuri M, Capanna R, Briccoli A, Perin S, et al. Neurologic deficit after resection of the sacrum. Chir Organi Mov. 1997; 82(4): 357-72.

7. Nakai S, Yoshizawa H, Kobayashi S, Maeda K, Okumura Y. Anorectal and bladder function after sacrifice of the sacral nerves. Spine (Phila Pa 1976). 2000; 25(17): 2234-9.

8. Gunterberg B, Norlén L, Stener B, Sundin T. Neurologic evaluation after resection of the sacrum. Invest Urol. 1975; 13(3): 183-8.

9. Ohata N, Ozaki T, Kunisada T, Morimoto Y, Tanaka M, Inoue H. Extended total sacrectomy and reconstruction for sacral tumor. Spine (Phila Pa 1976). 2004; 29(6): E123-6.

10. Randall RL, Bruckner J, Lloyd C, Pohlman TH, Conrad EU 3rd. Sacral resection and reconstruction for tumors and tumor-like conditions. Orthopedics. 2005; 28(3): 307-13.

11. Sahakitrungruang C, Chantra K, Dusitanond N, Atittharnsakul P, Rojanasakul A. Sacrectomy for primary sacral tumors. Dis Colon Rectum. 2009; 52(5): 913-8.

12. Sar C, Eralp L. Surgical treatment of primary tumors of the sacrum. Arch Orthop Trauma Surg. 2002; 122(3): 148-55.

13. Wuisman P, Lieshout O, Sugihara S, van Dijk M. Total sacrectomy and reconstruction: oncologic and functional outcome. Clin Orthop Relat Res. 2000; 381: 192-203. 